\title{
Laparoscopic Evaluation Shows Deficiencies in Memory Ring Deployment During Small Ventral Hernia Repair
}

\author{
Christophe R. Berney
}

Published online: 29 June 2011

(c) Société Internationale de Chirurgie 2011

\section{To the Editor}

I congratulate Frederik Berrevoet and collaborators for their recent publication in World Journal of Surgery [1]. The repair of small ventral hernias in 28 patients was performed with a self-expanding, memory-containing reinforcement polypropylene/expanded polytetrafluoroethylene (ePTFE) patch and objectively assessed by simultaneous laparoscopic evaluation. The results were very disappointing, showing inadequate positioning of the hernia patch at laparoscopy in the majority of the cases and a $14.8 \%$ recurrence rate after a median follow-up of 25 months. There are few points that I would like to raise.

Repair of small umbilical and ventral hernias based on the open underlay technique has become very popular as it is quick and easy to perform, offering the benefits of having the prosthesis placed behind the abdominal wall, while at the same time avoiding an unnecessary, more invasive, and costly laparoscopic approach. Unfortunately, the downside is the potential risk of developing intraperitoneal meshrelated complications, such as formation of dense adhesions or mesh erosion into the small bowel. This happened in one of their patient only two weeks postoperatively, and therefore was most likely related to a technical error.

I believe that the size of the hernia patch is the main limitation in this approach. The larger the patch, the higher the risk of getting "cupping" of the mesh as the positioning straps are pulled up for fixation. Such cupping increases the exposure of the polypropylene side of the mesh.

C. R. Berney $(\square)$

Department of Surgery, Bankstown-Lidcombe Hospital,

University of N.S.W, 67 Rickard Road, Bankstown,

NSW 2200, Australia

e-mail: berneycr@hotmail.com
In their study, Berrevoet et al. used a large hernia patch ( $8 \mathrm{~cm}$ in diameter) in $86 \%$ of their cases and a mediumsized patch $(6.4 \mathrm{~cm})$ for the remaining procedures. In comparison, over the last 4 years I have used the identical prosthesis on 66 patients, but a large size mesh in only $15 \%$ of the cases (unpublished data). Although these hernia repairs were not controlled laparoscopically, I did not encounter any recurrence or major complication at a median follow-up of 20 months. A recent study, also using smaller size hernia patches, showed similar results [2].

Nevertheless, in light of the findings of Berrevoet et al., and to conclude, I recommend placing the mesh in the retromuscular or preperitoneal space rather than intraperitoneally. Should that placement not be practicable, my next suggestion would be to avoid using the large size prosthetic patch, unless it is possible to secure its edge safely to the abdominal wall, thus avoiding the above-described cupping effect.

\section{References}

1. Berrevoet F, Van den Bossche B, de Baerdemaeker L, de Hemtinne B (2010) Laparoscopic evaluation shows deficiencies in memory ring deployment during small ventral hernia repair. World J Surg 34:1710-1715. doi:10.1007/s00268-010-0600-7

2. Martin DF, Williams RF, Mulrooney T, Voeller GR (2008) Ventralex mesh in umbilical/epigastric hernia repairs: clinical outcomes and complications. Hernia 12:379-383 